\title{
Registration of a Curve on a Surface Using Differential Properties
}

\author{
Alexis Gourdon, Nicholas Ayache \\ INRIA - Project Epidaure - 2004 Route des Lucioles, BP 93 \\ 06902 Sophia Antipolis Cedex, France. \\ Email : alexis.gourdon@sophia.inria.fr
}

\begin{abstract}
This article presents a new method to find the best rigid registration between a curve and a surface. It is possible to write a compatibility equation between a curve point and a surface point, which constrains completely the 6 parameters of the sought rigid displacement. This requires the local computation of third order differential quantities and leads to an algebraic equation of degree 16 .

A second approach consists in considering pairs of curve and surface points. Then only first order differential are necessary to compute locally the parameters of the rigid displacement. Although computationally more expensive, the second approach is more robust, and can be accelerated with a preprocessing of the surface data.

To our knowledge, it is the first method which takes full advantage of local differential computations to register a curve on a surface.
\end{abstract}

\section{Introduction}

Finding the best spatial registration between a rigid curve and a rigid surface is an important problem in the medical field when a volume medical image must be registered either with a single cross-section acquired later with a CT-Scanner or MRI, or with a 3D curve acquired with a laser range finder on the external surface of a patient. Besl mentionned this problem in a paper on $3 \mathrm{D}$ registration [1], and Grimson presented recently an industrial application of this type but restricted to the recognition of cylinder objects [2].

The scope of our study is more general in that we do not restrict the shape of the observed surfaces or curves. In fact, although results are presented with planar curves, the developped formalism is valid also for general spatial curves and free form surfaces. On the other hand, we assume that it is possible to compute the differential properties of both the curve and the surface, either up to the third order (first approach), or at least up to the first order (second approach). Both are reasonable assumptions with high resolution medical volume images, where adequate spatial filtering allows for the extraction of anatomical surfaces and curves, with the computation of differential properties $[3,4,5]$.

\section{Registration Using one Point on Curve and Surface}

\subsection{Geometrical Constraints}

At each point of a parametric curve, we can define an intrinsic orthonormal frame $(\mathbf{t}, \mathbf{n}, \mathbf{b})$ (the Frenet frame) and metric invariants (the curvature $k$ and torsion $\tau$ ). We can also build at each point of a parametric surface the two fundamental forms and infer from them an intrinsic orthonormal frame $\left(\mathbf{e}_{\mathbf{1}}, \mathbf{e}_{\mathbf{2}}, \mathbf{N}\right)$ (the principal frame) and the two principal curvatures. $\left(k_{1}, k_{2}\right)$ ) [6]

When a curve lies on a surface, its tangent vector is in the tangent plane of the surface. We can then also construct a third intrinsic orthonormal frame $(t, g, N)$, 
called the Darboux frame. Thus, for each of these frames, we can express the derivatives of the frame along the curve with respect to the arc length $s$ in the same frame (moving frame method) [6]. Using the relations between the Darboux frame and the Frenet frame or the principal frame we obtain:

$$
\begin{array}{rlrl}
k_{n} & =k \cos \theta= & k_{1} \cos ^{2} \varphi+k_{2} \sin ^{2} \varphi & \text { (normal curvature) } \\
k_{g}=k \sin \theta=\frac{1}{k_{1}-k_{2}}\left(\frac{\partial k_{1}}{\partial e_{2}} \cos \varphi+\frac{\partial k_{2}}{\partial e_{1}} \sin \varphi\right)+\frac{d \varphi}{d s} & \text { (geodesic curvature) }{ }^{1} \\
\tau_{g}=\tau-\frac{d \theta}{d s} & = & \left(k_{1}-k_{2}\right) \cos \varphi \sin \varphi & \text { (geodesic torsion) }
\end{array}
$$

The knowledge of $\theta$ (angle betwen the normal to the curve and the normal to the surface) and $\varphi$ (angle between the curve tangent and the principal direction $\left.\mathbf{e}_{1}\right)$ characterizes the rotation between the Frenet frame and the principal frame.

For each point on the curve, we can compute its curvature $k$ and its torsion $\tau$ and their derivatives with respect to the arc length $s$. Moreover, for each point of the surface the principal curvatures $\left(k_{1}, k_{2}\right)$ and their derivatives along $\mathbf{e}_{\mathbf{1}}$ and e 2 can be computed. However $k_{n}, k_{g}, \tau_{g}$, the two angles $\theta, \varphi$ and their derivatives with respect to $s$ are unknown. In our problem, we have a model $S$ of a surface and a curve $\alpha$ that we wish to register on $S$. For every point $m$ of $\alpha$ and its homologous point $M$ on the surface, every pair $(\theta, \varphi)$ which is solution of $(1,2,3)$ gives us a unique registered Frenet frame. It is then easy to find the rotation of the Frenet frame and to infer the rigid transformation which maps $m$ into $M$.

\subsection{Determining the Angles $\theta$ and $\varphi$}

The drawback of the system $(1,2,3)$ where the unknown values are $(\theta(s), \varphi(s))$ is the presence of the derivatives of $(\theta(s), \varphi(s))$ with respect to $s$ (except for (1)). Thus, in order to find $(\theta, \varphi)$ we must eliminate those derivatives. By derivating (1) with respect to $s$ and using $(2,3)$ and ${ }^{2}$ we obtain:

$\frac{1}{k} \frac{d k}{d s} k_{n}+k_{g}\left(3 \tau_{g}-\tau\right)=\frac{\partial k_{1}}{\partial e_{1}} \cos ^{3} \varphi+3 \frac{\partial k_{1}}{\partial e_{2}} \cos ^{2} \varphi \sin \varphi+3 \frac{\partial k_{2}}{\partial e_{1}} \cos \varphi \sin ^{2} \varphi+\frac{\partial k_{2}}{\partial e_{2}} \sin ^{3} \varphi=C(\varphi)$

Therefore we obtain one single algebraic equation in $\varphi$ with

$$
f(\varphi)=\left(k^{2}-k_{n}^{2}(\varphi)\right)\left(3 \tau_{g}(\varphi)-\tau\right)^{2}-\left(C(\varphi)-\frac{1}{k} \frac{d k}{d s} k_{n}(\varphi)\right)^{2}=0
$$

Since (5) is an algebraic equation of degree sixteen, there is no hope to find explicit roots for the general case, but classical methods may be used [7].

For each solution $\varphi,(1)$ gives us $\cos \theta ; \sin \theta$ is obtained by (2) and (4).

\subsection{Matching Algorithm}

First at all we have to compute differential invariants in volume or planar images. The use of a Gaussian convolution filter transforms the digital 3D image into an infinitely differentiable function $I(x, y, z)[3,5]$. Then the implicit function theorem allows us to express the differential caracteristics of the iso-surface $I(x, y, z)=a$ as expressions of the derivatives of $I(x, y, z)[4]$.

Now we can describe our matching algorithm:

${ }^{1}$ for each fonction $M(u, v)$ defined on the surface, $\frac{\partial M}{\partial e_{1}}(m)=\frac{d M}{d t}\left(m+t e_{1}\right)$ for $(t=0)$ is called the derivative of $M$ with respect to $e_{1}$ in $m$.

${ }^{2}$ for each function $P$ of the coordinates of the surface $(u, v)$, the derivative of the restriction of $P$ on the curve may be written: $\frac{d P}{d s}=\frac{\partial P}{\partial e_{1}} \cos \varphi+\frac{\partial P}{\partial e_{2}} \sin \varphi$ 
1. For each remaining pair of points with one point on the curve and the other one on the surface, solve the equation for the angle $\varphi$. Then for each root:

(a) Compute $\theta$ and determine the rigid transformation.

(b) Apply the tranformation to the curve.

(c) If a sufficient number of curve points lie at a distance of the surface smaller than a preset threshold, store the 6 parameters of the rigid transformation in a hash-table.

2. Repeat until at least one bucket of the hash-table contains a sufficient number of stored transfomations.

By applying the estimated transformation to the curve we can easily eliminate irrelevant transformations. However this last process requires the computation, for each point of the curve, of the closest point on the surface. This can be done by using octree-spline [8]. As soon as the initial guess is reasonably good, we can improve the registration by using an iterative method (see [9] for instance). Unfortunately, to find the initial guess, we have to compute third order derivatives on the curve and on the surface and solve an algebraic equation of high degree. This makes the method applicable to high quality data only. To address this last point, we present now a more expensive but more robust approach.

\section{Registration Using pair of Points}

\subsection{Geometrical Constraints}

The geometric constraints between a pair of points $(a, b)$ on the curve $\alpha$ and its homologous pair of points $(A, B)$ on the surface $S$ require only first order differential invariants on the curve and surface.

Since the tangent to a curve lying on a surface is in the tangent plane of the surface and that the scalar product is invariant by a rigid transformation, the constraints between the pairs $(a, b)$ et $(A, B)$ can be written as:

$$
\left\{\begin{array} { l } 
{ \| \mathbf { a b } \| = \| \mathbf { A B } \| = d } \\
{ \langle D ( \mathbf { t } _ { \mathbf { a } } ) | \mathbf { N } _ { \mathbf { A } } \rangle = \langle D ( \mathbf { t } _ { \mathbf { b } } ) | \mathbf { N } _ { \mathbf { B } } \rangle = 0 }
\end{array} \quad \text { and } \quad \left\{\begin{array}{l}
\left\langle D\left(\mathrm{t}_{\mathbf{a}}\right) \mid \mathbf{A B}\right\rangle=\left\langle\mathbf{t}_{\mathbf{a}} \mid \mathbf{a b}\right\rangle \\
\left\langle D\left(\mathbf{t}_{\mathbf{b}}\right) \mid \mathbf{A B}\right\rangle=\left\langle\mathrm{t}_{\mathbf{b}} \mid \mathbf{a b}\right\rangle
\end{array}\right.\right.
$$

$D$ is the unknown displacement, $\left(\mathbf{t}_{\mathbf{a}}, \mathbf{t}_{\mathbf{b}}\right)$ are the tangents to the curve in $(a, b)$ and $\left(\mathbf{N}_{\mathbf{A}}, \mathbf{N}_{\mathbf{B}}\right)$ are the normals to the surface in $(A, B)$ homologous points of $(a, b)$ by $D$. Writing $\left(\mathbf{N}_{\mathbf{A}}\left|\mathbf{N}_{\mathbf{B}}\right\rangle=\cos \Gamma\right.$ and expressing $\left(D\left(\mathbf{t}_{\mathbf{a}}\right), D\left(\mathbf{t}_{\mathbf{b}}\right)\right)$ in the bases of the tangent planes of the surface in $(A, B)^{3}$ we have:

$$
\left\{\begin{array}{l}
\sin \Gamma D\left(\mathrm{t}_{\mathbf{a}}\right)=\cos \alpha \mathbf{N}_{\mathbf{A}} \wedge \mathbf{N}_{\mathbf{B}}+\sin \alpha\left[\cos \Gamma \mathbf{N}_{\mathbf{A}}-\mathbf{N}_{\mathbf{B}}\right] \\
\sin \Gamma D\left(\mathrm{t}_{\mathbf{b}}\right)=\cos \beta \mathbf{N}_{\mathbf{A}} \wedge \mathbf{N}_{\mathbf{B}}+\sin \beta\left[\cos \Gamma \mathbf{N}_{\mathbf{B}}-\mathbf{N}_{\mathbf{A}}\right]
\end{array}\right.
$$

$(\alpha, \beta)$ are the angles between $\left(D\left(\mathbf{t}_{\mathbf{a}}\right), D\left(\mathbf{t}_{\mathbf{b}}\right)\right)$ and $\mathbf{N}_{\mathbf{A}} \wedge \mathbf{N}_{\mathbf{B}}$.

By writing $\mathbf{A B}$ in the two bases and by computing the scalar products $\left\langle D\left(\mathbf{t}_{\mathbf{a}}\right) \mid \mathbf{A B}\right\rangle$ and $\left\langle D\left(\mathbf{t}_{\mathbf{b}}\right) \mid \mathbf{A B}\right\rangle$ we get two equations for the angles $(\alpha, \beta)$ of the form $A \cos \theta+B \sin \theta=C^{4}$. As the scalar product is invariant we can write: $\left\langle D\left(\mathbf{t}_{\mathbf{a}}\right) \mid D\left(\mathbf{t}_{\mathbf{b}}\right)\right\rangle=\left\langle\mathbf{t}_{\mathbf{a}} \mid \mathbf{t}_{\mathbf{b}}\right\rangle=\cos \gamma$. Thus we have one more equation:

$$
\cos \alpha \cos \beta-\cos \Gamma \sin \alpha \sin \beta=\cos \gamma
$$

Then by solving the equations verified by $\alpha$ and $\beta$ and keep the roots which verify (6) we can find the rigid transformation which maps $(a, b)$ on $(A, B)$.

\footnotetext{
${ }^{3}$ when $\mathbf{N}_{\mathbf{A}} \wedge \mathbf{N}_{\mathbf{B}} \neq 0$

${ }^{4}$ An equation of the form $A \cos \theta+B \sin \theta=C$ has two roots if and only $A^{2}+B^{2} \geq C^{2}$
} 


\subsection{Specific Situations}

When $\sin \gamma=0$ and $\sin \Gamma \neq 0$, (i.e. the tangent vectors in $(a, b)$ are parallel). By using (6) we prove that $(\alpha, \beta)$ must be equal to 0 or $\pi$ and that $\left(\mathbf{t}_{\mathbf{a}}, \mathbf{t}_{\mathbf{b}}, \mathbf{N}_{\mathbf{A}} \wedge\right.$ $\mathbf{N}_{\mathbf{B}}$ ) are proportional vectors. This can be seen in the following equation:

$$
\lambda^{2}=\left(\frac{\left\langle\mathbf{N}_{\mathbf{A}} \wedge \mathbf{N}_{\mathbf{B}} \mid \mathbf{A B}\right\rangle}{\sin \Gamma}\right)^{2}=\left\langle\mathbf{a b} \mid \mathbf{t}_{\mathbf{a}}\right\rangle^{2}=\left\langle\mathbf{a b} \mid \mathbf{t}_{\mathbf{b}}\right\rangle^{2}
$$

Thus in this particular case we have two invariants on the pair of curve points and on the pair of surface points : the intrinsic distance $d$ and the quantity $\lambda$.

\subsection{Registration Algorithm}

Using the above-mentioned constraints, the matching algorithm is as follows:

1. for each pair of curve points and for each pair of surface points being at the same distance $d$ :

(a) find $\alpha$ and $\beta$ and keep them if they satisfy (6) with a given accuracy $\epsilon$.

(b) Compute for each solution $(\alpha, \beta)$ the corresponding rigid transformation. 2. Then proceed the same way as in the first algorithm.

The main advantage of this algorithm with respect to the one based on the computation of $\varphi$ comes from the use of first order differential invariants (curve tangents and surface normals), instead of the third order differential invariants.

However, this algorithm has a higher complexity: it is necessary to search for pairs of curve and scene points being at a similar distance of each other. A brute force algorithm leads to a combinatorial explosion, which is in $O\left(n^{2} p^{2}\right)$ ).

The complexity can be decreased by reducing the number of pairs of curve points, i.e. selecting those being at a sufficiently large distance of each other. The surface can be preprocessed beforehand: pairs of surface points can be ordered by increasing distance $d$. Then, at recognition time, a given pair of curve points is compared to surface pairs of similar distance with a $O\left(\log \left(n^{2}\right)\right)$ algorithm.

A further reduction in the number of such pairs can be easily obtained when dealing with planar and closed curves, by imposing parallel tangent vectors. In this case, as showed above, it is easy to compute 2 intrinsic invariants at each curve and scene point ( $d$ and $\lambda$ computed with respect to curve point of same tangent vector). The number of pairs of curve points then reduces to $O(p)$, since for a given curve point there is a finite number of points on the curve with parallel tangents. Applying both strategies can reduce the recognition complexity to $O(p \log (n))$ for planar curves, and $O\left(p^{2} \log (n)\right)$ in the general case.

\section{Experimental Results}

\subsection{Synthetic Examples}

After the generation of synthetic volume images of a 3D object (Fig. 1), we extracted the object surface for a given iso-intensity value [4], and a curve in a randomly selected 2D cross-section, for the same iso-intensity value. The surface had about 7000 points while the curve had about 150 points.

Using the first approach, and using the proposed third order differential invariants, (5) yielded from 0 to 12 solutions for $\varphi$. By computing the derivatives analytically, the program returned for $90 \%$ of the curve points, the correct $\varphi$ with an accuracy better than 5 degrees. When the derivatives were computed by local gaussian filtering this percentage was $75 \%$ of the curve points.

The second approach yielded a correct rigid transformation, for all our trials. In fact, we got additional solutions, which are quite reasonable due to the symmetries of the original object as can be seen in Fig. 1 . 

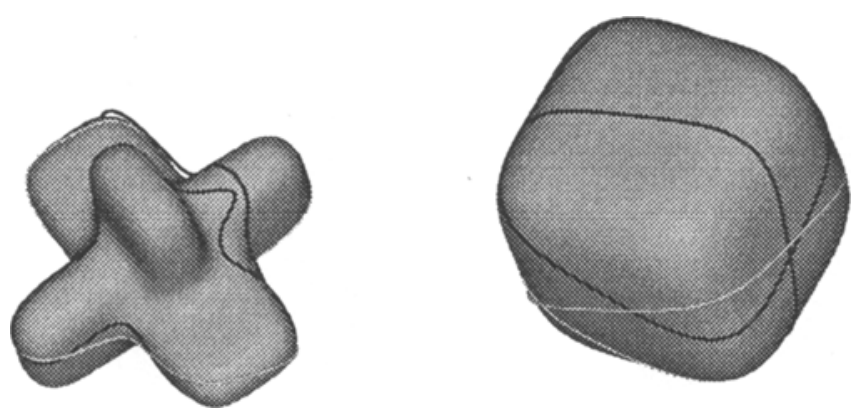

Fig. 1. Registrations on synthetic examples.

\subsection{Real Data}

We used a volume image of a skull acquired by an X-ray CT-Scan (provided by GE-MS), and we computed the iso-surface corresponding to the bone surface. In another image of the same skull in a different position, we extracted a single cross section and the iso-intensity contour corresponding again to the bone limit.

The algorithm using the second approach found the rigid transformation which superimposes the curve on the surface. As we knew that the cross section had been extracted grossly at the level of the orbits, we reduced the complexity of the matching algorithm by selecting only about 10 cross-sections of the first volume image of the skull, centered about the orbits and by taking the pairs of curve points whose inter-distance was larger than $75 \%$ of the curve diameter.

An iterative registration algorithm can improve the found solution. Typically, the initial solution is found with a tolerated distance of about 5 voxels between the transformed curve and the surface, and this distance can be decreased to 0.5 voxel after a few tens of iterations of the iterative closest point algorithm.

\section{Conclusion}

In this paper we have presented the differential constraints which can be exploited to register rigidly a curve on a surface. These constraints apply by considering either homologous points, which requires the computation of third order differential invariants and leads to a $O(n p)$ algorithm, or homologous pairs of points between the curve and the surface, which brings about the computation of first order differential invariants and leads to a brute force complexity of $O\left(n^{2} p^{2}\right)$ algorithm. This last complexity can be significantly reduced, typically to $O(p \log (n))$ by applying additional constraints, in particular when the curve is planar and closed.

We presented results both with synthetic and real data, showing that only the second algorithm is robust enough in the presence of noise. Anyhow, the constraints used in the first algorithm can be used efficiently during the verification stage of the second algorithm, making the whole study useful in practice.

\section{Aknowledgements}

The authors wish to thank J.P. Thirion for his contribution of the general formulation of the problem, J. Feldmar for stimulating discussions, G. Medioni and also G. Malandain for their useful comments. This research was partially supported by Digital Equipment Corporation. 

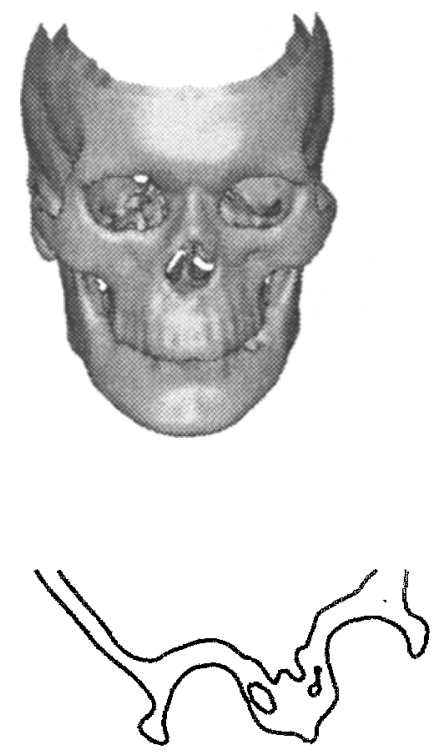
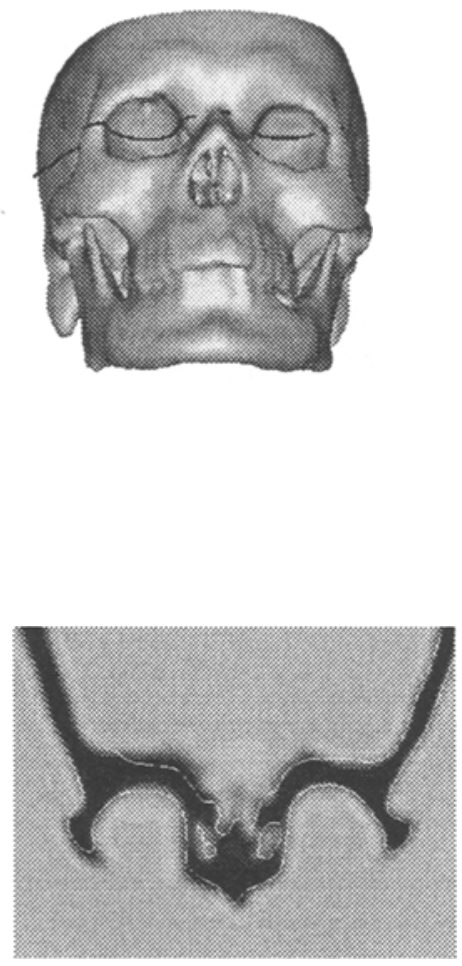

Fig. 2. Top: left: surface from the first CT scan; left: one particular fit. Bottom: left: curve from the second CT scan; right: the curve is superimposed on the interpolated cross section image.

\section{References}

1. P.J. Besl. The Free-Form Surface Matching Problem (Machine Vision for 3-D Scenes). H. Freeman, Ed., 1990.

2. W.E.L Grimson, T. Lozano-Pérez, N. Noble, and S.J. White. An automatic tube inspection system that finds cylinders in range data. In Proceedings CVPR '98, New York City, NY. IEEE, June 1993.

3. O. Monga, S. Benayoun, and O.D. Faugeras. Using partial derivatives of 3d images to extract typical surface features. In Proceedings CVPR 'gQ, Urbana Champaign, Illinois. IEEE, July 1992.

4. Jean-Philippe Thirion and Alexis Gourdon. The 3D marching lines algorithm and its application to crest lines extraction. Technical Report 1672, INRIA, May 1992.

5. B.H. Romeny, L. Florack, A. Salden, and M. Viergever. Higher order differential structure of images. In H.H. Barrett and A.F. Gmitro, editors, IPMI, pages 77-93, Flagstaff, Arizona (USA), June 1993. Springer-Verlag.

6. M.P. Do Carmo. Differential geometry of curves and surfaces. Prentice Hall, 1976.

7. William H. Press, Brian P. Flannery, Saul A. Teukolsky, and William T. Vetterling. Numerical Recipes in C, The Art of Scientific Computing. Cambridge University Press, 1990.

8. Stéphane Lavallée, Richard Szeliski, and Lionel Brunie. Matching 3-d smooth surfaces with their 2-d projections using 3-d distance maps. In SPIE, Geometric Methods in Computer Vision, San Diego, Ca, July 1991.

9. P.J. Besl and N.D. McKay. A method for registration of 3-D shapes. IEEE Transactions on PAMI, 14(2), February 1992. 